\title{
La revista Anuario de Estudios Americanos analizada desde las bases de datos documentales: evolución temática, distribución de la autoría e impacto/
}

\author{
The Journal Anuario de Estudios Americanos Analyzed from \\ the Bibliographic Databases: Thematic Evolution, \\ Distribution of Authorship and Impact
}

\author{
Luis Rodríguez Yunta \\ https://orcid.org/0000-0002-8424-6205 \\ Centro de Ciencias Humanas y Sociales, CSIC
}

Se realiza un análisis de la revista Anuario de Estudios Americanos en el contexto de las publicaciones españolas de Historia de América. El estudio se basa en su presencia en bases de datos documentales y en los datos que pueden extraerse de ÍnDICEs-CSIC, Web of Science y Scopus. Se valora la evolución temática, la distribución de la autoría y el impacto de esta revista en comparación con otros títulos de la disciplina.

Palabras clave: Revistas Académicas; Revistas de Historia; Estudios Latinoamericanos; Bases de Datos; Análisis Bibliométrico.

An analysis of the journal Anuario de Estudios Americanos is carried out in the context of the Spanish publications of Latin American History. The study is based on its presence in bibliographic databases and in the data that can be extracted from ÍnDICEs-CSIC, Web of Science and Scopus. The thematic evolution, the distribution of the authorship and the impact of this journal are valued in comparison with other titles of the discipline.

Keywords: Academic Journals; Journals of History; Latin American Studies; Databases; Bibliometric analysis.

Copyright: (C) 2018 CSIC. Este es un artículo de acceso abierto distribuido bajo los términos de la licencia de uso y distribución Creative Commons Reconocimiento 4.0 Internacional (CC BY 4.0). 


\section{Presencia de Anuario de Estudios Americanos en bases de datos y plataformas de revistas}

La revista científica desempeña una función esencial en el sistema de comunicación de la ciencia, ${ }^{1}$ ya que además de ser una herramienta de difusión de conocimiento se le atribuye un papel de certificación o validación de la actividad investigadora. La edición de publicaciones periódicas de prestigio es un servicio que las instituciones científicas de mayor relevancia asumen entre sus funciones para beneficio de la comunidad nacional e internacional de investigadores. Por ello resulta relevante analizar las características editoriales e indicios de calidad de una publicación en el contexto de su área temática.

Anuario de Estudios Americanos es una revista publicada desde 1944 por el Consejo Superior de Investigaciones Científicas (CSIC) desde la Escuela de Estudios Hispano-Americanos en Sevilla. ${ }^{2}$ Como publicación especializada en Historia de América puede encuadrarse tanto en el conjunto de las publicaciones de Estudios Históricos como en el área transversal de los Estudios Latinoamericanos. El americanismo español tiene connotaciones específicas por las evidentes relaciones históricas, culturales, políticas, sociales y económicas entre España y América Latina. ${ }^{3}$ En este contexto, la Historia de América es un campo consolidado en nuestro país que está representado por diferentes publicaciones específicas. La existencia de varias revistas de la especialidad, con prestigio y difusión internacional, es un reflejo de su importancia y de la implicación de un considerable número de investigadores. Es notable que el propio CSIC edite además una segunda publicación específica de Historia de América, Revista de Indias, gestionada desde el Instituto de Historia en Madrid.

En cierta medida, puede decirse que Anuario de Estudios Americanos ha representado a la escuela sevillana de estudios americanistas. Las publicaciones periódicas científicas nacidas a mediados de siglo XX se creaban con el objetivo principal de difundir las investigaciones realizadas en el centro editor. Progresivamente estas revistas han ido abandonando este rol endogámico para convertirse en publicaciones cada vez más abiertas a la participación de autores de cualquier universidad o centro de investigación.

1 Para un panorama de la evolución de las revistas científicas, su situación actual y principales tendencias, véanse las contribuciones de diferentes autores en Abadal, 2017.

2 Bernabéu y Varela, 2010.

3 González Martínez y Carrera Troyano, 2010. 
Los artículos que se publican son evaluados y seleccionados, pero lógicamente las revistas dependen de que los autores envíen sus textos. En consecuencia, la calidad de los contenidos está muy relacionada con el prestigio y capacidad de cada revista para captar el interés por publicar de los autores de mayor relevancia en la disciplina.

Su presencia en bases de datos es un elemento hoy indispensable para su prestigio y difusión a nivel internacional. Ello repercute tanto en la consulta y citación de sus artículos como en la incorporación de nuevos autores que desean remitir sus textos. Anuario de Estudios Americanos está presente tanto en buscadores académicos como en bases de datos bibliográficas, repositorios, recolectores, portales y directorios de revistas. ${ }^{4}$ Sin embargo, la cobertura varía en los diferentes sistemas de información en los que se localiza (tabla 1).

La revista en versión impresa está disponible en numerosas bibliotecas universitarias en todo el mundo, pero los usuarios demandan hoy en día el acceso telemático a los textos en su versión electrónica. Para la versión en texto completo, la fuente principal es la propia plataforma de la Editorial CSIC que realiza la edición electrónica en acceso abierto. ${ }^{5}$ Esta iniciativa se puso en marcha en 2007 y ha ido incorporando la versión digitalizada de años anteriores, pero de momento solo cubre los números publicados desde 1994. ${ }^{6}$ Para la consulta de los primeros 50 años en formato digital es necesario acudir a un producto comercial (Periodicals Index Online - Periodicals Archive Online). ${ }^{7}$ Además, en 1997 se editó un cd-rom con la edición íntegra de los volúmenes I al LIII-1. ${ }^{8}$

Los artículos de Anuario de Estudios Americanos son analizados de forma sistemática por buscadores académicos y bases de datos bibliográficas, aunque también con coberturas variables que se recogen en la ta-

4 Para un panorama actualizado de las fuentes de información disponibles en Internet para la investigación histórica, véase Chaín-Navarro, 2017.

5 Revistas CSIC, Edición electrónica de las revistas científicas del CSIC: http://revistas.csic.es/.

6 Sarabia Viejo, 2010.

7 PIO Periodicals Index Online es una base de datos bibliográfica que recoge fondo antiguo de publicaciones periódicas de Humanidades y Ciencias Sociales. Está comercializada por la empresa ProQuest. Cuenta con un fichero paralelo, PAO Periodicals Archive Online, para los artículos que han sido digitalizados. Para los usuarios españoles su consulta es posible en muchas bibliotecas universitarias o en la red de bibliotecas del CSIC.

8 Anuario de Estudios Americanos/Escuela de Estudios Hispano-Americanos, Universidad Internacional de Andalucía, Fundación El Monte, Fundación Histórica Tavera, Madrid, Digibis Fundación Histórica Tavera, 1997, 1 disco compacto; contiene los volúmenes I al LIII.1, años 1944-1996. 
bla 1. A través de ellos, estos artículos pueden aparecer en los resultados de búsquedas realizadas en productos que recogen la producción española $(\text { InDICEs-CSIC })^{9}$ o hispánica (Dialnet),${ }^{10}$ en recolectores de datos de revistas electrónicas $(R E D I B, D O A J),{ }^{11}$ en productos bibliográficos especializados (Historical Abstracts, HLAS, HAPI) ${ }^{12}$ y en índices de citas (Web of Science, Scopus). ${ }^{13}$

Además de estos productos que realizan un análisis sistemático de esta publicación, hay otros productos bibliográficos que realizan un seguimiento selectivo, como America History \& Life, Anthropological Index Online, Bibliografía de la Literatura Española y MLA International Bibliography. ${ }^{14}$

9 InDICEs-CSIC, Información y Documentación de la Ciencia en España, es un portal bibliográfico disponible en línea desde 2018: https://indices.csic.es/. Integra las anteriores bases ISOC, ICYT e IME, productos pioneros en la puesta en marcha de las primeras bases de datos en España y que recogen la producción científica de las revistas españolas desde la década de 1970.

10 Dialnet es un portal bibliográfico que recoge publicaciones científicas de todo el mundo, con especial atención al ámbito hispánico: https://dialnet.unirioja.es/. El proyecto fue iniciado en 2002 por la Biblioteca de la Universidad de La Rioja y desde 2009 está gestionado por la Fundación Dialnet.

11 REDIB, Red Iberoamericana de Innovación y Conocimiento Científico, es una plataforma de revistas creada por el CSIC y Universia: https://www.redib.org/. DOAJ, Directory of Open Access Journals, es una plataforma puesta en marcha por la Universidad de Lund a partir de 2003: https://doaj.org/. En ambos casos se trata de recolectores o agregadores de contenidos, que incorporan de forma automatizada registros bibliográficos solamente de revistas científicas de acceso abierto.

12 Historical Abstracts, es una base de datos bibliográfica especializada en Historia moderna y contemporánea de todo el mundo, excepto Estados Unidos y Canadá; fue creada por la empresa ABCClio y actualmente es producida por Ebsco. HAPI, Hispanic American Periodicals Index, es una base de datos especializada en Estudios Latinoamericanos gestionada desde UCLA: http://hapi.ucla.edu/. HLAS, Handbook of Latin American Studies Online, es otro producto bibliográfico especializado en Latinoamérica elaborado por la Library of Congress: http://lcweb2.loc.gov/hlas/.

13 Web of Science es una plataforma que agrupa diferentes bases de datos bibliográficas, índices de citas e informes de indicadores bibliométricos creados inicialmente por el ISI Institute of Scientific Information y gestionados actualmente por Clarivate Analytics. Contiene un fichero específico para Humanidades, Arts \& Humanities Citation Index, si bien sus datos no se incluyen en los informes bibliométricos (JCR Journal Citation Reports). Scopus es un producto competidor elaborado por Elsevier, cuyos datos son utilizados para elaborar indicadores bibliométricos en varias fuentes: CiteScore metrics, CWTS Journals Indicators y Scimago Journal \& Country Rank. En España el acceso a las plataformas Web of Science y Scopus para instituciones públicas de investigación se gestiona desde la FECYT y pueden consultarse en https://www.recursoscientificos.fecyt.es/.

14 En estos productos solo se incluyen las referencias de los artículos relacionados con su especialidad temática: America History \& Life (elaborado por ABC-Clio y especializado en Historia de Estados Unidos y Canadá), Anthropological Index Online (Royal Anthropological Institute, artículos de antropología), Bibliografía de la Literatura Española (creada por Carmen Simón Palmer y gestionada actualmente por ProQuest) y MLA International Bibliography (Modern Language Association, artículos sobre Literatura internacional). 


\begin{tabular}{|l|l|l|}
\hline \multicolumn{1}{|c|}{$\begin{array}{c}\text { Base de datos } \\
\text { o Plataforma }\end{array}$} & Cobertura & \multicolumn{1}{c|}{ Características } \\
\hline Revistas CSIC & $1994-2018$ & Texto completo (plataforma editorial). \\
\hline $\begin{array}{l}\text { Periodicals Index Online } \\
\text { - Periodicals Archive } \\
\text { Online }\end{array}$ & $1944-2000$ & Texto completo (fondo histórico). \\
\hline ÍnDICEs-CSIC & $1974-2018$ & $\begin{array}{l}\text { Referencias bibliográficas con enlaces al } \\
\text { texto completo desde 1994. }\end{array}$ \\
\hline Dialnet & $1974-2018$ & $\begin{array}{l}\text { Referencias bibliográficas con enlaces al } \\
\text { texto completo desde 1999. }\end{array}$ \\
\hline REDIB & $1994-2018$ & $\begin{array}{l}\text { Referencias bibliográficas con enlaces al } \\
\text { texto completo desde 1994. }\end{array}$ \\
\hline DOAJ & $1994-2018$ & $\begin{array}{l}\text { Referencias bibliográficas con enlaces al } \\
\text { texto completo desde 1994. }\end{array}$ \\
\hline Historical Abstracts & $1953-2018$ & $\begin{array}{l}\text { Referencias bibliográficas, base de datos } \\
\text { especializada en Historia. }\end{array}$ \\
\hline HAPI & $1976-2018$ & $\begin{array}{l}\text { Referencias bibliográficas, base } \\
\text { de datos especializada en Estudios } \\
\text { Latinoamericanos. }\end{array}$ \\
\hline $\begin{array}{l}\text { Arts \& Humanities } \\
\text { Science) }\end{array}$ & $2008-2018$ & $\begin{array}{l}\text { Referencias bibliográficas, base } \\
\text { de datos especializada en Estudios } \\
\text { Latinoamericanos. }\end{array}$ \\
\hline Scopus & $1975-2013$ algunos años anteriores. \\
\hline HLAS & Indeb & $\begin{array}{l}\text { Índice de citas. Parcialmente incluye } \\
\text { algunos años anteriores. }\end{array}$ \\
\hline
\end{tabular}

Tabla 1. Bases de datos y Plataformas en las que está presente Anuario de Estudios Americanos. Elaboración propia.

En la valoración de características editoriales que realiza la red iberoamericana Latindex,,$^{15}$ Anuario de Estudios Americanos cumple la totalidad de los 33 criterios de calidad en la edición impresa y 35 sobre 36 en

15 Latindex, Sistema Regional de Información en Línea para Revistas Científicas de América Latina, el Caribe, España y Portugal, es una red iberoamericana coordinada desde la UNAM. Puede consultarse en http://latindex.org/latindex/inicio. 
la versión electrónica, por lo que en ambos casos la publicación entra en la sección de Catálogo. Las características de calidad definidas por esta red se basan en buenas prácticas en los procesos de edición científica. En el momento de su implantación había graves carencias en las revistas de Humanidades, ${ }^{16}$ pero actualmente su cumplimiento es generalizado.

La propia presencia en bases de datos se utiliza a menudo como indicador de la calidad o prestigio de las publicaciones. Entre las fuentes que valoran especialmente este indicador, Anuario de Estudios Americanos se sitúa en la categoría A en Ciencias Humanas en la Clasificación $C I R C^{17}$ y tiene un ICDS con el nivel máximo de 11 en MIAR. ${ }^{18}$ Otro sistema de categorización de revistas en el que ha sido seleccionado es ERIH Plus. ${ }^{19}$

La revista forma parte de la selección de fuentes utilizada en los índices de citas de Web of Science, pero su productor no publica datos estadísticos de factor de impacto u otros indicadores para las revistas de Humanidades. Por ello, como indicadores estándares elaborados a partir de citas, solo podemos contar con las fuentes bibliométricas basadas en los datos de Scopus. En CiteScore Rank de 2017, Anuario de Estudios Americanos ocupa la posición 527 entre 771 revistas en la categoría Cultural Studies (tercer cuartil). Igualmente se encuentra en el tercer cuartil de esta categoría en Scimago Journal Rank de 2017, si bien estuvo situada en el segundo cuartil en 2014, 2015 y 2016. La revista también figuró en los proyectos de análisis de citas de revistas españolas, $R E S H$ e $I n-R e c h,{ }^{20}$ pero estos sistemas de información no están vigentes actualmente. Igualmente, figura en Journal Scholar Metrics ${ }^{21}$ en donde ocupa la posición 583 sobre 701 revistas inter-

16 Román Román, Vázquez Valero y Urdín, 2002.

17 CIRC Clasificación Integrada de Revistas Científicas: https://www.clasificacioncirc.es/. En su versión actual, presentada en 2016, es un producto elaborado por la spin-off EC3metrics de Granada.

18 MIAR Matriz de Información para el Análisis de Revistas: http://miar.ub.edu/. En esta plataforma puede consultarse el ICDS (Índice Compuesto de Difusión Secundaria) basado en la presencia en bases de datos científicas.

19 ERIH Plus, European Reference Index for Humanities and Social Sciences, es una iniciativa de la European Science Foundation para crear un índice de revistas académicas europeas de calidad en Ciencias Sociales y Humanidades. Actualmente está gestionado por el NSD, organismo público de Noruega.

20 RESH, Sistema de valoración integrada de revistas españolas de ciencias sociales y humanas, fue elaborado por el grupo EPUC del CSIC. Con datos de impacto del periodo 2000 a 2008. In-Rech, Índice de impacto de las revistas españolas de ciencias de humanidades, fue elaborado por el grupo EC3 de la Universidad de Granada con datos de 2004 a 2008.

21 Journal Scholar Metrics es un proyecto desarrollado también por el Grupo EC3 de la Universidad de Granada, basado en las citas recogidas por el buscador académico Google Scholar en su portal Google Scholar Metrics: http://www.journal-scholar-metrics.infoec3.es/. 
nacionales de Historia (cuarto cuartil) según las citas recogidas por Google Scholar Metrics para el periodo 2010-2014.

Anuario de Estudios Americanos cuenta también con el Sello de Calidad de Revistas Científicas Españolas concedido por la Fundación Española para la Ciencia y la Tecnología (FECYT). Este certificado asegura que la publicación se encuentra entre las revistas científicas españolas de excelencia en su difusión internacional y en el cumplimiento de buenas prácticas de revisión y edición.

En la propuesta de categorización de las revistas españolas de Historia, realizada en la base de datos ISOC y basada en indicadores sobre trayectoria, apertura de la autoría e internacionalidad en el periodo 20042013, esta revista quedó encuadrada en la categoría principal. De 10 indicadores, mejoraba la media de la disciplina en 8 de ellos. Al aplicar estos mismos baremos sobre el conjunto de publicaciones españolas en Web of Science y/o Scopus, Anuario de Estudios Americanos figuraba en la posición 11 sobre 233 títulos analizados y mejoraba la media en 7 de 10 indicadores. ${ }^{22}$

Finalmente, en el informe elaborado por la red REDIAL sobre las revistas europeas especializadas en Estudios Latinoamericanos ${ }^{23}$ se destaca su posición como una de las publicaciones de mayor prestigio en el americanismo internacional. En un ámbito marcado por la juventud y falta de continuidad de gran número de iniciativas editoriales, puede considerarse como un título consolidado y de referencia en su especialidad.

\section{Análisis bibliométrico: metodología}

Para hacer un análisis bibliométrico de Anuario de Estudios Americanos se han extraído datos de InDICEs-CSIC, portal bibliográfico que incorpora los registros disponibles anteriormente en la base ISOC. Esta base de datos ofrece una cobertura amplia de la revista (1974-2018) y ofrece diferentes campos para el análisis: autoría, filiación institucional, clasificación de los artículos y temas tratados en cada artículo (descriptores de materias, identificadores y topónimos).

Con el objetivo de establecer algunas comparaciones se han extraído también de InDICEs-CSIC datos de otras revistas españolas especializadas

22 Rodríguez Yunta, 2014; 2015.

23 Rodríguez Yunta, Svensson y Manzano Fraile, 2016. 
en Historia de América. Para establecer los títulos a comparar se han seleccionado las revistas de esta especialidad presentes en la base de datos Scopus. Con ello se pretende comparar títulos similares en cuanto a temática y difusión. Estas publicaciones son: Boletín Americanista de la Universitat de Barcelona, Illes i Imperis de la Universitat Pompeu Fabra, Revista Complutense de Historia de América de la Universidad Complutense de Madrid, Revista de Indias del Instituto de Historia del CSIC en Madrid y Temas Americanistas de la Universidad de Sevilla. Con ello se puede hacer una comparativa entre publicaciones de tres de los principales puntos focales del americanismo en España: Madrid, Barcelona y Sevilla, con dos títulos de cada uno de ellos.

En InDICEs-CSIC los registros del año en curso se han completado con los artículos de este número en elaboración. Para otras revistas solo han podido incluirse parcialmente los artículos editados en 2018.

Los datos sobre citación se han extraído de Web of Science y de Scopus. Las búsquedas se han realizado en septiembre de 2018. Se ha descartado utilizar los datos de citas del buscador académico Google Scholar por considerar que este producto carece de la necesaria estabilidad y fiabilidad estadística que sí se presupone en las bases de datos bibliográficas.

\section{Análisis de la temática de los artículos publicados}

A partir de los registros de la revista en InDICEs-CSIC podemos analizar la evolución del enfoque temático de la revista en dos grandes apartados: periodo histórico (época precolombina, edad moderna y edad contemporánea) y ámbito geográfico (diferentes países). El análisis se circunscribe a los años recogidos en esta base de datos: 1974 a 2018.

El análisis de la distribución temática por periodos muestra un predominio general de los trabajos sobre Historia moderna (505 artículos), sobre los de Historia contemporánea (345 en total). En el gráfico 1 puede verse como a partir de 1987 los estudios de contemporánea se incrementan y superan a los de moderna en años determinados. Por el contrario los artículos sobre el periodo precolombino son muy escasos (tan solo siete entre 1974 y 2018). El cómputo se completa con otros 83 trabajos sobre periodos amplios o sobre la fase de transición entre los siglos XVIII y XIX. 


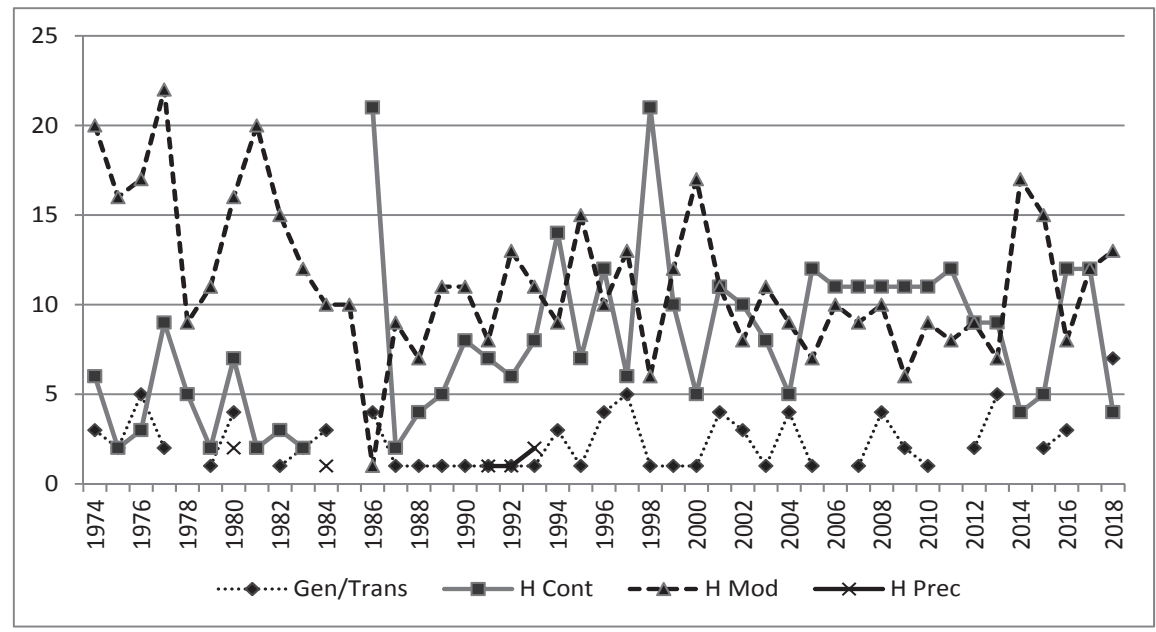

GRÁFICO 1. Número de artículos publicados por Anuario de Estudios Americanos entre 1974 y 2018, dedicados a Estudios generales o de transición (Gen/Trans), Historia contemporánea (H Cont), Historia moderna (H Mod) e Historia precolombina (H Prec). Fuente: ÍnDICEs-

CSIC. Elaboración propia.

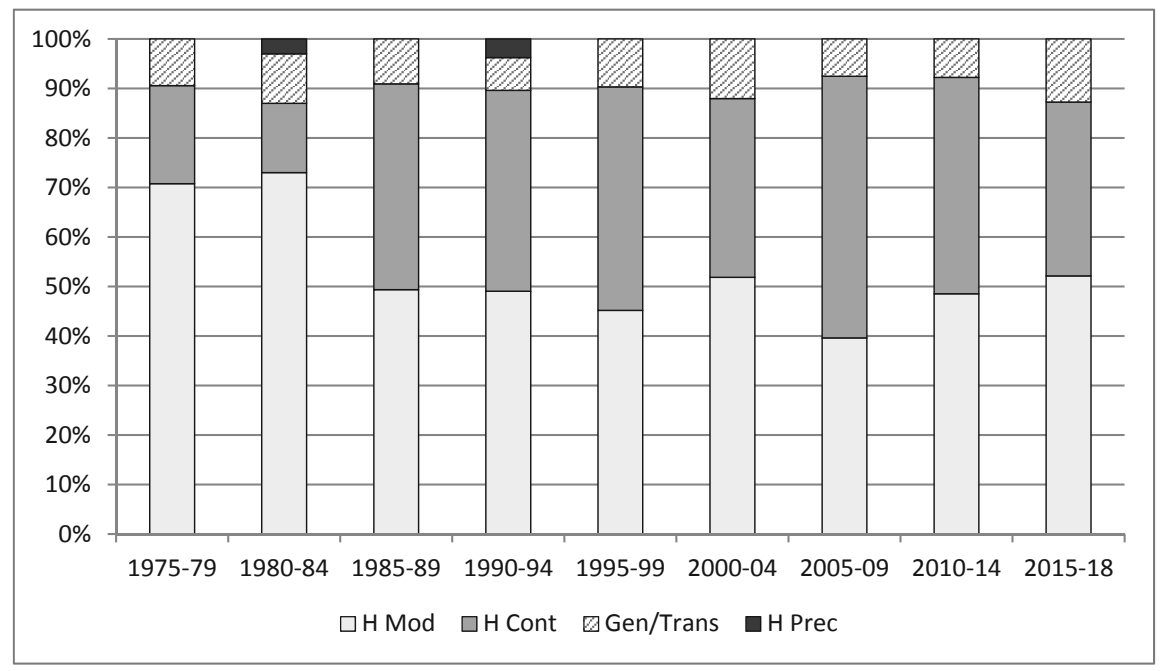

GráfICo 2. Porcentaje de artículos publicados por Anuario de Estudios Americanos por quinquenios entre 1974 y 2018, dedicados a Historia moderna (H Mod), Historia contemporánea (H Cont), Estudios generales o de transición (Gen/Trans) e Historia precolombina (H Prec). Fuente: ÍnDICEs-CSIC. Elaboración propia. 
Analizando esta evolución en porcentajes por quinquenios (gráfico 2) se muestra con mayor claridad esta evolución general:

- hasta 1984 la revista publicaba más de un $70 \%$ de sus trabajos en relación con la época moderna;

- a partir de 1985 la Historia contemporánea se incrementa y equilibra con los estudios de moderna aunque manteniéndose algo por debajo;

- entre 2005 y 2009 la Historia contemporánea se sitúa por encima de los trabajos de Historia moderna;

- a partir de 2010 se vuelve a una situación de cierto equilibrio con predominio de los trabajos de Historia moderna.

Para comparar esta distribución temática con otras revistas españolas de Historia de América se analiza el periodo 2000-2018. Con ello se pretende reflejar la situación actual de estas publicaciones en el siglo XXI. En el gráfico 3 destaca que la Historia moderna predomina solamente en las publicaciones editadas en Sevilla, Anuario de Estudios Americanos y Temas Americanistas, en las que supone un $48 \%$ y un $52 \%$ respectivamente. Por el contrario los estudios sobre Historia contemporánea son muy superiores en las publicaciones de Madrid y Barcelona, especialmente en Boletín Americanista $(69 \%)$.

Como puede verse en el gráfico 3, la escasa presencia de trabajos sobre época prehispánica es una constante en las revistas españolas de Historia de América. En el caso de la Universidad Complutense de Madrid este hecho está justificado por la edición de otro título centrado en estudios americanistas antropológicos, etnohistóricos y arqueológicos: la Revista Española de Antropología Americana. ${ }^{24}$ Por el contrario, no existe una publicación especializada en este ámbito en Barcelona o Sevilla.

En relación con los países objeto de estudio, en el cómputo general de 1974 a 2018 la atención se dirige principalmente hacia México (169 trabajos), Argentina (152), Perú (129) y Cuba (85). En una posición intermedia se encuentran Chile (44), Ecuador (37), Colombia (36), Bolivia (33) y Venezuela (30). Con menor número de estudios se sitúan Filipinas (26), Puerto Rico (23), Brasil (20), República Dominicana (19), Estados Unidos (18), Guatemala (16), Uruguay (15), Paraguay (11), Panamá (9), Canadá (5), Nicaragua (5), Haití (4), Honduras (4) o El Salvador (1).

24 Sánchez Montañés e Iglesias Ponce de León, 2002. 


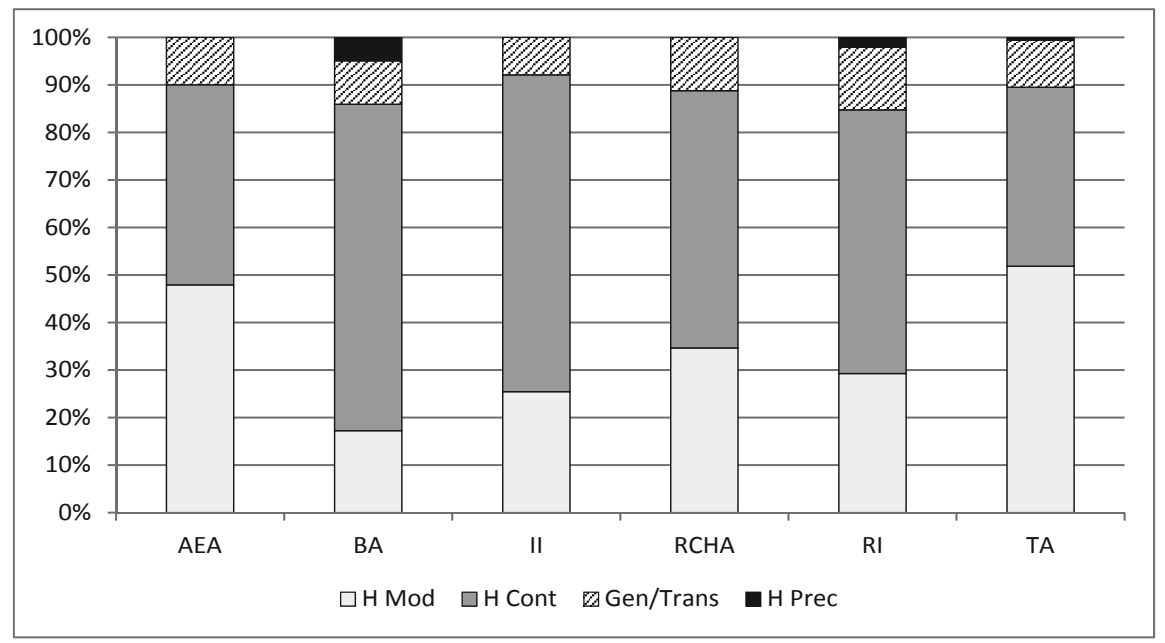

GrÁfICO 3. Porcentaje de artículos entre 2000 y 2018 dedicados a Historia moderna (H Mod), Historia contemporánea (H Cont), Estudios generales o de transición (Gen/ Trans) e Historia precolombina (H Prec), en las revistas españolas: Anuario de Estudios Americanos (AEA), Boletín Americanista (BA), Illes i Imperis (II), Revista Complutense de Historia de América (RCHA), Revista de Indias (RI) y Temas Americanistas (TA). Fuente: ÍnDICEs-CSIC. Elaboración propia.

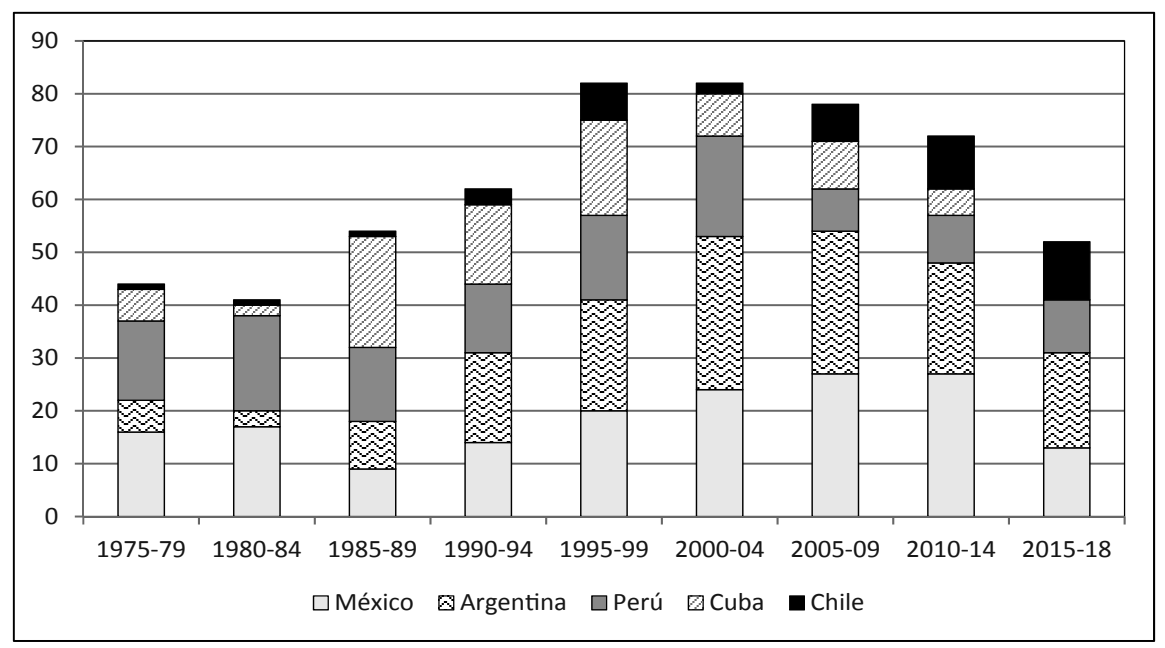

GráfICO 4. Número de artículos publicados por Anuario de Estudios Americanos entre 1974 y 2018, dedicados a los países más tratados: México, Argentina, Perú, Cuba y Chile. Fuente: ÍnDICEs-CSIC. Elaboración propia. 
Al analizar la evolución por quinquenios de los trabajos sobre los cinco países más estudiados (gráfico 4) se aprecian algunas diferencias de interés:

- México es el país con mayor número de estudios, pero es sobrepasado en algunos periodos por Perú (1980-1989), Cuba (1985-1994) y Argentina (1990-2004 y 2015-2018);

- los trabajos sobre Argentina se incrementaron notablemente a partir de 1990;

- los estudios sobre Cuba ocuparon un lugar importante entre 1985 y 1999 con el centenario de su independencia, pero se han reducido notablemente a partir sobre todo de 2010;

- los trabajos sobre Perú se han reducido de forma importante al disminuir los estudios de Historia moderna, especialmente desde 2005.

\begin{tabular}{|l|l|l|l|}
\hline \multicolumn{1}{|c|}{$1980-1989$} & \multicolumn{1}{|c|}{$1990-1999$} & \multicolumn{1}{c|}{$2000-2009$} & \multicolumn{1}{c|}{ 2010-2018 } \\
\hline Esclavitud (21) & Sociedad colonial & Sociedad colonial & Indígenas (25) \\
Abolicionismo & $(26)$ & $(31)$ & Sociedad colonial \\
$(17)$ & Indígenas (20) & Indígenas (30) & $(18)$ \\
Población (13) & Comercio colonial & Historiografía (17) & Comercio colonial \\
Arte colonial (12) & $(17)$ & Conflictos sociales & $(17)$ \\
Esclavos (10) & Independencia & $(16)$ & Independencia \\
Comerciantes (9) & hispanoamericana & Mujeres (13) & hispanoamericana \\
Comercio colonial & $(14)$ & Administración de & $(16)$ \\
$(9)$ & Comerciantes (13) & justicia (12) & Comerciantes (10) \\
Datos biográficos & Historiografía (12) & Iglesia Católica & Fronteras (10) \\
$(9)$ & Economía colonial & $(11)$ & Zonas fronterizas \\
Impuestos (8) & $(11)$ & Intelectuales (11) & $(9)$ \\
Indígenas (8) & Mujeres (10) & Zonas fronterizas & Administración \\
& Pensamiento & $(11)$ & colonial (8) \\
& político (10) & Comercio colonial & Colonización (8) \\
& Relaciones & $(9)$ & Expediciones \\
navales (8)
\end{tabular}

TABLa 2. Diez descriptores de materias más utilizados en cada década para caracterizar los temas tratados por los artículos de Anuario de Estudios Americanos en la base de datos ÍnDICEs CSIC. Elaboración propia. 
Otro dato que podemos extraer de ÍnDICEs CSIC es la evolución de los descriptores de materias. ${ }^{25}$ En la tabla 2 se presentan los diez descriptores más utilizados para la descripción de los temas tratados en los artículos en cada década. Mientras que en la década de 1980 el foco de atención se dirigió hacia la esclavitud, a partir de 1990 predominan los estudios sobre sociedad colonial e indígenas. Algunos temas como los procesos de independencia o los estudios de género tienen una aparición más irregular, están muy presentes en algunas décadas, pero ausentes en otras.

\section{Análisis de la autoría}

De los registros de la revista en InDICEs-CSIC podemos analizar la relación de autores para el periodo 1974-2018. En total hay 756 firmas diferentes para 952 artículos. La diversidad en la autoría (relación entre firmantes y artículos) está proporcionada para una publicación de Humanidades, dado que en estas disciplinas predominan los trabajos de un único autor. En Anuario de Estudios Americanos se han editado en este periodo 878 trabajos con una sola firma (92\% del total), 64 con dos autores (7 \%), siete casos con tres, dos con cuatro y uno con cinco. En la tabla 3 se analiza la distribución del número de artículos por autor.

A partir de los registros de la revista en InDICEs-CSIC podemos analizar también la evolución de la procedencia de los autores. En este caso limitamos el análisis a los artículos publicados a partir de 1993, fecha en la que Anuario de Estudios Americanos incorpora sistemáticamente el dato sobre la filiación institucional de los autores. ${ }^{26}$ En el periodo 1993-2018 las instituciones españolas con mayor número de trabajos han sido la Universidad de Sevilla (67 trabajos) y el CSIC (44), seguidas de la Universidad Complutense de Madrid (17), Universitat de Barcelona (10), Universidad de Granada (8), Universidad de Valladolid (8), Universidad del País Vasco (8), Universitat Autònoma de Barcelona (7), Universidad de Córdoba (6) y Universidad de Huelva (6)

25 Se analizan en este sentido los términos añadidos por los documentalistas del CSIC en los registros de la base de datos para describir los temas tratados en cada documento, no las palabras clave utilizadas por los autores.

26 Para años anteriores hay algunos artículos que sí incluyen el dato de la filiación de los autores pero no se recoge esta información en la mayor parte de los casos, por lo que se inicia el análisis en 1993. 


\begin{tabular}{|c|c|c|c|}
\hline $\begin{array}{l}\text { Frecuencia en } \\
\text { la autoría }\end{array}$ & Número de autores & $\begin{array}{l}\text { Porcentaje } \\
\text { de autores }\end{array}$ & $\begin{array}{c}\text { Porcentaje de } \\
\text { artículos }^{27}\end{array}$ \\
\hline $\begin{array}{l}\text { Autores con } \\
5 \text { o más artícu- } \\
\text { los }\end{array}$ & $\begin{array}{l}11 \text { casos: Castañeda Delgado, } \\
\text { Paulino (5); Ferrer Muñoz, Ma- } \\
\text { nuel (5); Gil, Juan (11); Hampe } \\
\text { Martínez, Teodoro (6); Heredia, } \\
\text { Edmundo A. (6); Iwasaki Cauti, } \\
\text { Fernando (5); Langue, Frédéri- } \\
\text { que (6); Mena García, Carmen } \\
\text { (7); Navarro García, Luis (7); } \\
\text { Santamaría García, Antonio (5) } \\
\text { y Vila Vilar, Enriqueta (5) }\end{array}$ & $1 \%$ & $7 \%$ \\
\hline $\begin{array}{l}\text { Autores con } \\
4 \text { artículos }\end{array}$ & 17 casos & $2 \%$ & $7 \%$ \\
\hline $\begin{array}{l}\text { Autores con } \\
3 \text { artículos }\end{array}$ & 36 casos & $5 \%$ & $11 \%$ \\
\hline $\begin{array}{l}\text { Autores con } \\
2 \text { artículos }\end{array}$ & 97 casos & $13 \%$ & $20 \%$ \\
\hline $\begin{array}{l}\text { Autores con } \\
1 \text { artículo }\end{array}$ & 595 casos & $79 \%$ & $63 \%$ \\
\hline
\end{tabular}

TABLA 3. Distribución de número de artículos publicados por el mismo autor en Anuario de Estudios Americanos 1974-2018. Fuente: ÍnDICEs-CSIC.

Elaboración propia.

De los 582 artículos publicados desde 1993, solamente trece son colaboraciones entre autores de diferentes instituciones. De ellas siete son entre organizaciones españolas, cinco entre una institución española y una extranjera (dos con Cuba y uno con Bolivia, Chile y Perú) y finalmente un caso entre centros de otros países (Estados Unidos y Perú).

Aunque las dos principales instituciones representadas en Anuario de Estudios Americanos, Universidad de Sevilla y CSIC, destacan con claridad sobre las restantes, el índice de endogamia es muy bajo. Al analizar el porcentaje que representan estas instituciones sobre el total de artículos (gráfico 5) se aprecia que entre ambas no superan el $30 \%$ de los trabajos publicados, y no llega en ningún periodo al $15 \%$ para el caso de la entidad editora (CSIC).

27 La columna de porcentaje de artículos suma más de un $100 \%$ debido a que en los artículos firmados por varios autores están contabilizados tantas veces como firmas. 


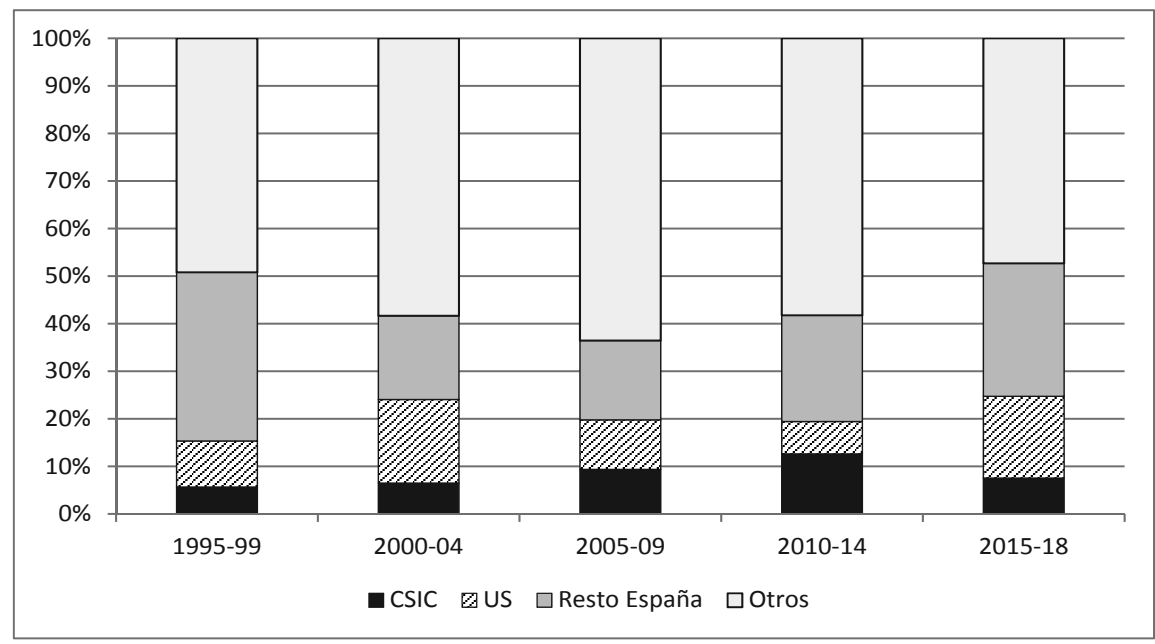

Gráfico 5. Porcentaje de artículos publicados en Anuario de Estudios Americanos por quinquenios entre 1995 y 2018, firmados por autores del CSIC y de la Universidad de Sevilla (US), frente a otras instituciones españolas y de otros países. Fuente: ÍnDICEs-CSIC. Elaboración propia.

El porcentaje de artículos firmados por investigadores de la entidad editora en Anuario de Estudios Americanos para el total del periodo analizado es de solo el $8 \%$, el más bajo entre las revistas españolas de Historia de América que se han analizado en este trabajo (véase tabla 4).

\begin{tabular}{|l|r|r|r|r|r|c|c|}
\hline Revistas & CSIC & $U S$ & $U C M$ & $U B$ & $U P F$ & $\begin{array}{c}\text { Total } \\
\text { artículos }\end{array}$ & $\begin{array}{c}\text { Porcen. } \\
\text { Editor }\end{array}$ \\
\hline AEA & $\mathbf{4 9}$ & 69 & 17 & 10 & 3 & 582 & $\mathbf{8} \%$ \\
\hline BA & 1 & 2 & 12 & $\mathbf{1 0 2}$ & 2 & 387 & $\mathbf{2 6 \%}$ \\
\hline II & 3 & 1 & 0 & 3 & $\mathbf{3 7}$ & 127 & $\mathbf{2 9} \%$ \\
\hline RCHA & 15 & 4 & $\mathbf{7 2}$ & 6 & 0 & 296 & $\mathbf{2 4} \%$ \\
\hline RI & $\mathbf{9 4}$ & 19 & 28 & 13 & 5 & 666 & $\mathbf{1 4 \%}$ \\
\hline TA & 4 & $\mathbf{5 3}$ & 1 & 6 & 0 & 186 & $\mathbf{2 8 \%}$ \\
\hline
\end{tabular}

TABLA 4. Número de artículos de las entidades editoras entre 1993 y 2018 en las revistas españolas: Anuario de Estudios Americanos (AEA), Boletín Americanista (BA), Illes $i$ Imperis (II), Revista Complutense de Historia de América (RCHA), Revista de Indias (RI) y Temas Americanistas (TA). Se aporta porcentaje correspondiente al valor de la entidad editora (destacado en negrita) sobre el total de artículos publicados en cada caso.

Fuente: ÍnDICEs-CSIC. Elaboración propia. 
La aportación de trabajos firmados por autores de filiaciones extranjeras se sitúa en el $55 \%$ de los artículos en el periodo 1993-2018. Destacan especialmente las aportaciones firmadas por autores de Argentina (109 trabajos), México (44), Chile (28), Francia (27), Estados Unidos (21), Perú (16) y Venezuela (12).

\begin{tabular}{|l|c|c|}
\hline Países latinoamericanos & Autores & Tema de estudio \\
\hline Argentina & 109 & 125 \\
\hline México & 44 & 117 \\
\hline Chile & 28 & 38 \\
\hline Perú & 16 & 65 \\
\hline Venezuela & 12 & 22 \\
\hline Brasil & 8 & 15 \\
\hline Cuba & 6 & 49 \\
\hline Uruguay & 6 & 14 \\
\hline Bolivia & 4 & 26 \\
\hline República Dominicana & 4 & 10 \\
\hline Ecuador & 3 & 19 \\
\hline Colombia & 3 & 18 \\
\hline Costa Rica & 0 & 0 \\
\hline El Salvador & 0 & 1 \\
\hline Guatemala & 0 & 9 \\
\hline Haití & 0 & 3 \\
\hline Honduras & 0 & 3 \\
\hline Nicaragua & 0 & 4 \\
\hline Paraguay & 0 & 7 \\
\hline Panamá & 0 & 6 \\
\hline Puerto Rico & 0 & 10 \\
\hline
\end{tabular}

TABLa 5. Comparativa de la presencia de países latinoamericanos en Anuario de Estudios Americanos 1993-2018, por filiación institucional de los autores y como tema tratado en los artículos. Fuente: ÍnDICEs-CSIC. Elaboración propia. 
Lógicamente, hay una cierta relación entre los países con mayor presencia de autores y los países latinoamericanos más tratados, si bien en algunos casos la mayor parte de los estudios proceden de investigadores españoles, europeos o norteamericanos. En la tabla 5 puede verse cómo el interés por Argentina o Chile tiene una mayor base en la aportación de historiadores de estos países que en los casos de México, Perú, Cuba o Bolivia.

\begin{tabular}{|l|r|r|r|r|r|r|}
\hline Autores extranjeros & $A E A$ & $B A$ & $I I$ & $R C H A$ & $R I$ & $T A$ \\
\hline Argentina & 109 & 89 & 12 & 34 & 74 & 29 \\
\hline México & 44 & 55 & 6 & 28 & 68 & 23 \\
\hline Chile & 28 & 6 & 0 & 10 & 24 & 16 \\
\hline Francia & 27 & 9 & 8 & 4 & 36 & 1 \\
\hline Estados Unidos & 21 & 5 & 9 & 7 & 40 & 0 \\
\hline Perú & 16 & 3 & 3 & 11 & 16 & 3 \\
\hline Venezuela & 12 & 9 & 1 & 1 & 9 & 2 \\
\hline Brasil & 8 & 17 & 2 & 13 & 27 & 0 \\
\hline Alemania & 7 & 4 & 2 & 2 & 13 & 0 \\
\hline Cuba & 6 & 1 & 4 & 2 & 13 & 5 \\
\hline Italia & 6 & 1 & 1 & 4 & 8 & 2 \\
\hline Uruguay & 6 & 3 & 1 & 4 & 3 & 4 \\
\hline Bolivia & 4 & 1 & 0 & 1 & 4 & 0 \\
\hline Portugal & 4 & 0 & 1 & 0 & 3 & 0 \\
\hline República Dominicana & 4 & 1 & 0 & 1 & 2 & 0 \\
\hline Suecia & 4 & 0 & 0 & 0 & 1 & 0 \\
\hline Colombia & 3 & 9 & 0 & 5 & 15 & 4 \\
\hline Ecuador & 3 & 0 & 1 & 0 & 1 & 1 \\
\hline Países Bajos & 2 & 1 & 1 & 1 & 2 & 1 \\
\hline Reino Unido & 0 & 2 & 1 & 1 & 10 & 0 \\
\hline Puerto Rico & & & 3 & 5 & 12 & 0 \\
\hline
\end{tabular}

TABLa 6. Número de artículos de autores extranjeros entre 1993 y 2018 en las revistas españolas: Anuario de Estudios Americanos (AEA), Boletín Americanista (BA), Illes i Imperis (II), Revista Complutense de Historia de América (RCHA), Revista de Indias (RI) y Temas Americanistas (TA). Se excluyen los países con menos de 5 artículos en la suma de todas las revistas. Fuente: ÍnDICEs-CSIC. Elaboración propia. 
Las revistas especializadas en Estudios Latinoamericanos tienen en general un alto grado de internacionalidad en la autoría. ${ }^{28}$ En un estudio sobre la revista América Latina Hoy, publicación americanista del ámbito de Ciencias Sociales editada por la Universidad de Salamanca, la participación de autores extranjeros es incluso mayor, del $75 \%$, especialmente con aportaciones desde Estados Unidos, México y Argentina. ${ }^{29}$ Igualmente, la mayor parte de las descargas de la versión electrónica en las revistas americanistas del CSIC provienen de Estados Unidos y Latinoamérica. ${ }^{30}$

Comparando con otras revistas españolas especializadas en Historia de América, puede observarse en la tabla 6 que el predominio de autores de Argentina y México es similar en todos los títulos, si bien en el caso de Anuario de Estudios Americanos, la aportación desde Argentina es especialmente importante. Por el contrario resulta menor la participación de autores de otros países como Alemania, Brasil, Cuba, Colombia, Estados Unidos, Francia, Reino Unido o Puerto Rico, en relación con otras publicaciones especialmente Revista de Indias.

\section{Análisis de datos de citación}

En Humanidades, y específicamente en el campo de la Historia, los tradicionales análisis de citación tienen un alcance limitado. Las referencias bibliográficas se dispersan sobre una amplia tipología de documentos y fuentes primarias. Escasamente incluyen artículos publicados en los últimos años y mantienen una vida media superior a los diez años. En un estudio sobre las citas presentes en Revista de Indias entre 1995 y 1999 un $62 \%$ se dirigían a monografías y solamente un $16 \%$ a artículos de revista. ${ }^{31}$

La plataforma Web of Science nos permite extraer datos de citación para los artículos de Anuario de Estudios Americanos a partir de 2008. En septiembre de 2018 recogía 232 artículos del periodo 2008-2018, para los que se obtuvo un promedio de 0,93 citas por artículo y un índice-h de 6 . En la tabla 7 se muestra la lista de las principales publicaciones de procedencia de las citas recogidas en esta plataforma. Aunque las autocitas de la propia

28 Román, Sorli Rojo y Giménez Toledo, 2006-2007.

29 Freidenberg y Trak Vásquez, 2012.

30 Rodríguez, Sastre y Gutiérrez, 2010.

31 Más Bleda y Chaín Navarro, 2004-2007. 
revista ocupan el primer lugar en la lista, su porcentaje es solamente de un $7 \%$, lo que puede considerarse dentro de la normalidad de las buenas prácticas científicas.

\begin{tabular}{|l|c|c|}
\hline Publicaciones citantes & Artículos citantes & Porcentaje \\
\hline Anuario de Estudios Americanos & 13 & $7,4 \%$ \\
\hline Revista de Indias & 11 & $6,3 \%$ \\
\hline Estudios Atacameños & 6 & $3,4 \%$ \\
\hline Historia Mexicana & 6 & $3,4 \%$ \\
\hline Colonial Latin American Review & 5 & $2,9 \%$ \\
\hline Geoforum & 5 & $2,9 \%$ \\
\hline Built Environment City Studies & 3 & $1,7 \%$ \\
\hline e-Spania Revue électronique d'études & 3 & $1,7 \%$ \\
\hline hispaniques médiévales & 3 & $1,7 \%$ \\
\hline Ethnohistory & 3 & $1,7 \%$ \\
\hline Historia Crítica & 3 & $1,7 \%$ \\
\hline Mélanges de la Casa de Velázquez & 3 & $1,7 \%$ \\
\hline Memoria Americana & 3 & $1,7 \%$ \\
\hline Mundo Agrario & 3 & $1,7 \%$ \\
\hline Seville: Through the Urban Void (Libro) & 2 & $1,1 \%$ \\
\hline Co-herencia & 2 & $1,1 \%$ \\
\hline Desacatos & 2 & $1,1 \%$ \\
\hline Hispania. Revista Española de Historia & 2 & $1,1 \%$ \\
\hline Hispanic Research. Journal of Iberian and & 2 & $1,1 \%$ \\
\hline Latin American Studies & 2 & $1,1 \%$ \\
\hline Historia Caribe & 2 & $1,1 \%$ \\
\hline Historia, Ciencias, Saude -Manguinhos & 2 & \\
\hline Magallánica: Revista de Historia Moderna & 2 & \\
\hline Revista Complutense de Historia de América & 2 & \\
\hline Revista Escuela de Historia & 2 & $1,1 \%$ \\
\hline Revista Tefros & 2 & \\
\hline Studia Historica. Historia Moderna & 2 & \\
\hline
\end{tabular}

Tabla 7. Publicaciones citantes de artículos de Anuario de Estudios Americanos. Se excluyen los títulos con una sola cita. Fuente: Web of Science. Elaboración propia. 
Entre las citas recogidas en Web of Science, el $71 \%$ proceden de publicaciones en español, pero también hay fuentes en inglés (26\%), portugués y francés. En el momento de la consulta (septiembre de 2018) el artículo más citado fue publicado en 2009: «Agua, poder y discursos: conflictos socio-territoriales por la construcción de centrales hidroeléctricas en la Patagonia chilena», de Hugo Romero Toledo, Hugo Romero Aravena y Ximena Toledo Olivares. La secuencia de citación refleja las dificultades para hacer estudios de impacto sobre los dos o tres primeros años en publicaciones de Humanidades, pues este artículo no recibió ninguna cita en los dos años siguientes a su publicación, teniendo sin embargo trece citas: tres en 2012, una en 2015, dos en 2016, cinco en 2017 y dos en 2018.

En el caso de Scopus los datos son algo menores al recoger datos de forma sistemática sobre esta revista solo a partir de 2010. Sobre 208 registros, se recogen 142 citas, con un índice-h de 4. En este recurso el artículo más citado es de 2011: «Luchas y defensas escondidas. Pluralismo legal y cultural como una práctica de resistencia creativa en la gestión local del agua en los andes», de Rutgerd Boelens, con siete menciones. Comparando con otros títulos españoles en la misma fuente, el índice $\mathrm{h}$ de Anuario de Estudios Americanos en el periodo 2010-2018 se encuentra algo por debajo de Revista de Indias pero por encima de los restantes casos (véase tabla 8).

\begin{tabular}{|l|c|c|c|c|}
\hline Revistas & Número de docs. & Citas recibidas & Índice $h$ & Art. más citado \\
\hline AEA & 208 & 142 & 4 & 7 \\
\hline BA & 168 & 54 & 3 & 7 \\
\hline II & 8 & 0 & 0 & 0 \\
\hline RCHA & 97 & 53 & 3 & 11 \\
\hline RI & 229 & 195 & 5 & 14 \\
\hline TA & 106 & 22 & 2 & 3 \\
\hline
\end{tabular}

TABLA 8. Número de documentos, citas recibidas, índice h y citas recibida por el artículo más citado entre 2000 y 2018 en las revistas españolas: Anuario de Estudios Americanos (AEA), Boletín Americanista (BA), Illes i Imperis (II), Revista Complutense de Historia de América (RCHA), Revista de Indias (RI) y Temas Americanistas (TA). Fuente: Scopus. Elaboración propia. 


\section{Conclusiones principales}

La presencia en bases de datos nacionales e internacionales y el análisis bibliométrico muestran que Anuario de Estudios Americanos es una publicación consolidada y de prestigio en el ámbito de los estudios de Historia de América. El grado de internacionalidad, la diversidad en la autoría, así como el hecho de estar seleccionada en las principales fuentes bibliográficas o haber obtenido el sello de calidad FECYT, certifican el cumplimiento de buenas prácticas editoriales.

Algunas características que se describen en este estudio son propias de las revistas de Humanidades: pocos trabajos en colaboración y escasas citas recibidas a sus artículos. Por otra parte, presenta otras características habituales en las publicaciones de Estudios Latinoamericanos: alta internacionalidad en la autoría e importancia de las consultas y citaciones desde Latinoamérica y Estados Unidos.

En comparación con otras publicaciones españolas de la especialidad, obtiene buenos resultados en el nivel de citas recibidas, aunque dentro de los niveles de baja citación de las revistas de Humanidades. Como indicador de calidad, cabe destacar que el porcentaje de artículos de investigadores de la entidad editora es el más bajo entre los títulos analizados. Por tanto, puede interpretarse que la endogamia es baja o está bien controlada en esta publicación.

Como características particulares frente a otras revistas españolas de Historia de América, destaca el mayor peso de los estudios de Historia moderna sobre los de contemporánea y la mayor participación de Argentina, tanto en la autoría como en los temas de estudio.

Recibido el 10 de septiembre de 2018

Aceptado el 17 de septiembre de 2018

\section{Referencias bibliográficas}

Abadal, Ernest (ed.), Revistas científicas. Situación actual y retos de futuro, Barcelona, Universitat, 2017.

Bernabéu, Salvador y Varela, Consuelo, «La Escuela de Estudios Hispano-Americanos y sus revistas: de la imprenta a Internet», Nuevo Mundo Mundos Nuevos, 2010, https://doi.org/10.4000/nuevomundo.59903 
Chaín-Navarro, Celia, «Fuentes para la Historia: Soportes, análisis, acceso y difusión en la era de Internet», en Gómez Díaz, R.; García Rodríguez, A. y Cordón García, J. A., Fuentes especializadas en Ciencias Sociales y Humanidades, Madrid, Pirámide, 2017.

Freidenberg, Flavia y Trak Vásquez, Juan Manuel, «América Latina Hoy, una evaluación de sus 20 años», América Latina Hoy, 60, 2012, 229-238, http:// revistas.usal.es/index.php/1130-2887/article/view/8990/9239

González Martínez, Elda Evangelina y Carrera Troyano, Miguel, «El Americanismo como punto central de la agenda política, social, intelectual y académica en España: apuntes explicativos», Anuario Americanista Europeo, 8, 2010, 21-38, http://www.red-redial.net/revista/anuario-americanista-europeo/article/viewFile/141/111

Más Bleda, Amalia y Chaín Navarro, Celia, «Análisis del uso de la producción científica a través de las citas realizadas en la Revista de Indias (1995-1999)», Contrastes, 13, 2004-2007, 195-213, http://revistas.um.es/contrastes/article/ view/84111

Rodríguez, Ramón B.; Sastre, Natividad y Gutiérrez, Cristina, «Revista de Indias y Anuario de Estudios Americanos. Visibilidad y uso de la edición electrónica», Nuevo Mundo Mundos Nuevos, 2010, https://doi.org/10.4000/nuevomundo.59766

Rodríguez Yunta, Luis, Categorización de las revistas españolas de Historia: Propuesta de indicadores sobre trayectoria, apertura de la autoría e internacionalidad en el periodo 2004-2013, Madrid, CCHS-CSIC, Documento de trabajo ISOC 2014/02, 2014, http://hdl.handle.net/10261/103402

Rodríguez Yunta, Luis, Categorización de las revistas españolas de ciencias humanas y sociales en Web of Science y/o Scopus: indicadores sobre trayectoria, apertura de la autoría e internacionalidad en el periodo 2004-2013, Madrid, CCHS-CSIC, Documento de trabajo ISOC 2015/12, 2015, http://hdl.handle. net/10261/116894

Rodríguez Yunta, Luis; Svensson, Anna y Manzano Fraile, Tomás, Revistas europeas de Estudios Latinoamericanos. Salamanca, Documento de Trabajo REDIAL 2016, http://hdl.handle.net/10261/134301

Román, Adelaida; Sorli Rojo, Ángela y Giménez Toledo, Elea, « ¿Tienen las revistas españolas de estudios latinoamericanos los niveles de internacionalización esperable, dado su ámbito de especialización?», Anuario Americanista Europeo, 4-5, 2006-2007, 425-440, http://www.red-redial.net/revista/anuario-americanista-europeo/article/viewFile/96/81

Román Román, Adelaida; Vázquez Valero, Manuela y Urdín Caminos, Carmen, «Los criterios de calidad editorial Latindex en el marco de la evaluación de las revistas españolas de humanidades y ciencias sociales», Revista Española de Documentación Científica, 25 (3), 2002, 286-307, https://doi.org/10.3989/ redc.2002.v25.i3.109 
Sánchez Montañés, Emma e Iglesias Ponce de León, María Josefa, «La visión del otro. Breve historia de la Revista Española de Antropología Americana», Revista de Dialectología y Tradiciones Populares, 57 (1), 2002, 59-70, https:// doi.org/10.3989/rdtp.2002.v57.i1.189

Sarabia Viejo, María Justina, «El Anuario y su edición digital», Nuevo Mundo Mundos Nuevos, 2010, https://doi.org/10.4000/nuevomundo.59683 